\title{
HACIA UNA TAXONOMÍA DE LAS ÁREAS METROPOLITANAS ESPAÑOLAS
}

\author{
José $\mathbf{M}^{\mathrm{a}}$ Feria Toribio \\ Departamento de Geografía, Historia y Filosofía. Universidad Pablo de Olavide \\ jmfertor@upo.es
}

\section{INTRODUCCIÓN}

El presente artículo aborda una propuesta de categorización de las áreas metropolitanas en España como expresión de la nueva escala, naturaleza y funcionamiento de su geografía urbana. Para ello, al carecer de un reconocimiento institucional e incluso estadístico de esta nueva realidad espacial, los análisis sobre los que se fundamenta la propuesta se basan en una definición homogénea y comparable internacionalmente de las áreas metropolitanas españolas. A partir de la misma, y centrando el foco en las áreas metropolitanas como procesos urbano-territoriales, frente a los más convencionales de jerarquías funcionales, los elementos y variables que se utilizan para establecer la categorización se refieren a las condiciones de organización, estructura y dinámicas internas de estos espacio metropolitanos.

Para afrontar el objetivo mencionado, cuatro serán los vectores fundamentales de aproximación a este complejo universo: tamaño, estructura, organización y dinámica, que a continuación son adjetivadas y explicadas. La primera, el tamaño, hace referencia obviamente a la dimensión cuantitativa del hecho urbano en cuestión, comúnmente expresada en volumen poblacional. Es el acercamiento más simple, y a veces el único, para una categorización de los elementos del sistema metropolitano, aunque aquí su principal contribución reside en el establecimiento inicial de la jerarquía de dicho sistema metropolitano. La estructura se entiende en esta aproximación como el sistema territorial de base, fundamentalmente la del poblamiento y la organización político-administrativa asociada a éste, constituyendo un factor fundamental para entender la concreta extensión, organización y dinámica de las áreas metropolitanas. No es necesario insistir en que es un error considerar que los procesos metropolitanos son uniformes, como si se desarrollaran sobre espacios en blanco; más bien al contrario se adaptan en sus patrones básicos a la concreta estructura del poblamiento de cada ámbito, introduciendo variantes significativas de organización metropolitana. Con respecto a la organización, se entiende ésta en términos funcionales y su reconocimiento se basa en los patrones de movilidad personal -la movilidad residencia-trabajo y la movilidad residencial-, en la medida en que estas reflejan de una manera sintética y conjunta las pautas de 
distribución de la residencia y del empleo y la interrelación espacial entre ambas. Por último, la dinámica se aborda de una forma relativamente elemental, a través de la evolución poblacional y a la espera de datos censales sobre movilidad o parque de viviendas que permita un análisis más en profundidad.

En la tabla adjunta se resumen de forma sintética los principales contenidos de la tarea realizada, con expresión de la taxonomía establecida, sus componentes y las características básicas de los elementos y variables de estructura, organización y dinámica sobre los que se fundamentado la categorización llevada a cabo, mientras que en los epígrafes siguientes se describen de forma breve cada una de ellas.

Tabla 1

CUADRO SÍNTESIS DE ELEMENTOS Y VARIABLES DE LAS CATEGORÍAS ESTABLECIDAS

\begin{tabular}{|c|c|c|c|c|c|}
\hline & COMPONENTES & TAMAÑOS & $\begin{array}{l}\text { ORGANIZACIÓN } \\
\text { FUNCIONAL }\end{array}$ & $\begin{array}{l}\text { ESTRUCTURA } \\
\text { TERRITORIAL }\end{array}$ & DINÁMICAS \\
\hline $\begin{array}{l}\text { REGIONES } \\
\text { METROPOLITANAS }\end{array}$ & $\begin{array}{l}\text { Madrid } \\
\text { Barcelona }\end{array}$ & $\begin{array}{l}\text { Rango de 4-6 } \\
\text { millones de } \\
\text { habitantes y } \\
70.000-120.000 \\
\text { has de suelo } \\
\text { artificializado } \\
\text { urbano (SAU) }\end{array}$ & $\begin{array}{l}\text { Organización } \\
\text { compleja } \\
\text { extendida a todo } \\
\text { el ámbito, con } \\
\text { centros y áreas } \\
\text { de organización } \\
\text { metropolitana } \\
\text { interna }\end{array}$ & $\begin{array}{l}\text { Más contenida } \\
\text { espacialmente } \\
\text { y con estructura } \\
\text { policéntrica } \\
\text { en Barcelona. } \\
\text { Estructuras más } \\
\text { difusas en Madrid, } \\
\text { con sistemas } \\
\text { reticulares y lineales }\end{array}$ & $\begin{array}{l}\text { Crecimiento } \\
\text { poblacional y en } \\
\text { menor medida y } \\
\text { expansión espacial. } \\
\text { Empieza a completar } \\
\text { el ciclo evolutivo } \\
\text { con procesos de } \\
\text { recentralización }\end{array}$ \\
\hline $\begin{array}{l}\text { AREAS } \\
\text { METROPOLITANAS } \\
\text { CONSOLIDADAS }\end{array}$ & $\begin{array}{l}\text { Valencia, Sevilla y } \\
\text { Bilbao }\end{array}$ & $\begin{array}{l}\text { Rango de } 1-2 \\
\text { millones de } \\
\text { habs. y } 20.000- \\
30.000 \text { has } \\
\text { SAU }\end{array}$ & $\begin{array}{l}\text { Organización } \\
\text { metropolitana } \\
\text { plenamente } \\
\text { consolidada con } \\
\text { existencia de } \\
\text { submercados de } \\
\text { vivienda y trabajo } \\
\text { en la corona }\end{array}$ & $\begin{array}{l}\text { Peso proporcional } \\
\text { entre centro y } \\
\text { corona en Valencia } \\
\text { y Sevilla; Bilbao, } \\
\text { una estructura } \\
\text { más difusa y } \\
\text { descentralizada }\end{array}$ & $\begin{array}{l}\text { Crecimiento } \\
\text { poblacional y en } \\
\text { menor medida y } \\
\text { expansión espacial } \\
\text { en Valencia y } \\
\text { Sevilla; estabilidad } \\
\text { en Bilbao. La } \\
\text { suburbanización se } \\
\text { ve acompañada por } \\
\text { redistribución en la } \\
\text { corona }\end{array}$ \\
\hline $\begin{array}{l}\text { AREAS } \\
\text { METROPOLITANAS } \\
\text { POLINUCLEARES }\end{array}$ & $\begin{array}{l}\text { Oviedo-Gijón- } \\
\text { Avilés; Bahía de } \\
\text { Cádiz-Jerez y } \\
\text { Málaga-Marbella }\end{array}$ & $\begin{array}{l}\text { Rango de } 0,5-1 \\
\text { millón de habs. } \\
\text { y } 15000-25000 \\
\text { has SAU }\end{array}$ & $\begin{array}{l}\text { Fusión de ámbitos } \\
\text { de organización } \\
\text { metropolitanas } \\
\text { claramente } \\
\text { individualizables }\end{array}$ & $\begin{array}{l}\text { Estructura } \\
\text { policéntrica en la Y } \\
\text { asturiana, reticular } \\
\text { en Bahía de Cádiz } \\
\text { Jerez y lineal- } \\
\text { dispersa en Málaga- } \\
\text { Marbella }\end{array}$ & $\begin{array}{l}\text { Tendencia hacía } \\
\text { la consolidación } \\
\text { de un sólo espacio } \\
\text { metropolitano } \\
\text { único. Dinámica } \\
\text { demográfica positiva } \\
\text { en las dos andaluzas; } \\
\text { estabilidad en la } \\
\text { asturiana }\end{array}$ \\
\hline
\end{tabular}




\begin{tabular}{|c|c|c|c|c|c|}
\hline & COMPONENTES & TAMAÑOS & $\begin{array}{l}\text { ORGANIZACIÓN } \\
\text { FUNCIONAL }\end{array}$ & $\begin{array}{l}\text { ESTRUCTURA } \\
\text { TERRITORIAL }\end{array}$ & DINÁMICAS \\
\hline $\begin{array}{l}\text { AREAS } \\
\text { METROPOLITANAS } \\
\text { STANDARD }\end{array}$ & $\begin{array}{l}\text { Las Palmas de GC } \\
\text { Zaragoza; Murcia; } \\
\text { Palma de M.; } \\
\text { Vigo; Granada; } \\
\text { A Coruña; Santa } \\
\text { Cruz de Tenerife; } \\
\text { Alicante; Donosti; } \\
\text { Valladolid; } \\
\text { Santander; } \\
\text { Tarragona; } \\
\text { Castellón y } \\
\text { Pamplona }\end{array}$ & $\begin{array}{l}\text { Rango de } \\
300.000- \\
800.000 \text { habs } \\
\text { y } 5.000-15000 \\
\text { has de SAU }\end{array}$ & $\begin{array}{l}\text { Áreas plenamente } \\
\text { consolidadas } \\
\text { como mercados } \\
\text { unitarios de } \\
\text { vivienda y trabajo, } \\
\text { con notable } \\
\text { prevalencia de } \\
\text { los procesos de } \\
\text { suburbanización, } \\
\text { aunque una } \\
\text { limitada } \\
\text { divesificación } \\
\text { de actividades } \\
\text { en las coronas } \\
\text { metropolitanas. }\end{array}$ & $\begin{array}{l}\text { El modelo estandar } \\
\text { de estructura } \\
\text { basada en una } \\
\text { ciudad central que } \\
\text { se suburbaniza en } \\
\text { los municipios del } \\
\text { entorno, queda } \\
\text { parcialmente diluido } \\
\text { en los casos de } \\
\text { Murcia y Zaragoza, } \\
\text { por el gran tamaño } \\
\text { y la estructura del } \\
\text { poblamiento en el } \\
\text { municipio central }\end{array}$ & $\begin{array}{l}\text { Tendencia general } \\
\text { a la expansión } \\
\text { espacial y cierta } \\
\text { diversificación } \\
\text { funcional en } \\
\text { la corona, } \\
\text { con tendencia } \\
\text { demográica } \\
\text { positiva. Las del } \\
\text { tercio nororiental, } \\
\text { más estables } \\
\text { demográficamente } \\
\text { y mayor equilibrio } \\
\text { funcional de } \\
\text { actividades }\end{array}$ \\
\hline $\begin{array}{l}\text { AREAS } \\
\text { METROPOLITANAS } \\
\text { MENORES }\end{array}$ & $\begin{array}{l}\text { Algeciras; } \\
\text { Almería; Girona; } \\
\text { Huelva; Ourense; } \\
\text { Logroño; Lleida; } \\
\text { Salamanca, León; } \\
\text { y Santiago de } \\
\text { Compostela. }\end{array}$ & $\begin{array}{l}\text { Rango entre } \\
200.000- \\
300.000 \\
\text { habitantes y } \\
2.000-5.000 \text { has } \\
\text { SAU }\end{array}$ & $\begin{array}{l}\text { Ámbitos } \\
\text { de potente } \\
\text { organización } \\
\text { como mercados } \\
\text { de trabajo pero } \\
\text { limitada en } \\
\text { la dimensión } \\
\text { residencial }\end{array}$ & $\begin{array}{l}\text { La ciudad central } \\
\text { tiene un peso } \\
\text { mayoritario en la } \\
\text { configuración del } \\
\text { ámbito excepto en } \\
\text { Girona y Santiago, } \\
\text { con estructuras } \\
\text { metropolitanas a } \\
\text { partir de una ciudad } \\
\text { central de relativo } \\
\text { pequeño tamaño }\end{array}$ & $\begin{array}{l}\text { Excepto en Ourense } \\
\text { y en menor } \\
\text { medida León y } \\
\text { Santiago, dinámicas } \\
\text { demográficas por } \\
\text { encima de la media. } \\
\text { Escaso indicios } \\
\text { de aparición } \\
\text { de procesos de } \\
\text { suburbanización, } \\
\text { salvo en Girona }\end{array}$ \\
\hline $\begin{array}{l}\text { AREAS } \\
\text { METROPOLITANAS } \\
\text { INCIPIENTES }\end{array}$ & $\begin{array}{l}\text { Córdoba, Elche, } \\
\text { Vitoria, Cartagena, } \\
\text { Burgos, Badajoz, } \\
\text { Albacete y Jaén } \\
\text { Manresa; El } \\
\text { Ferrol; Benidorm } \\
\text { y Pontevedra }\end{array}$ & $\begin{array}{l}\text { Rango entre } \\
100.000- \\
350.000 \text { habs. y } \\
1.000-6.000 \text { has } \\
\text { SAU }\end{array}$ & $\begin{array}{l}\text { Mercados locales } \\
\text { trabajo pero no de } \\
\text { vivienda }\end{array}$ & $\begin{array}{l}\text { Estructuras } \\
\text { mayoritarias de } \\
\text { municipios grandes } \\
\text { y cierto tamaño } \\
\text { poblacional que } \\
\text { asumen en su } \\
\text { interior la mayor } \\
\text { parte de los } \\
\text { procesos existentes } \\
\text { de suburbanización. } \\
\text { El otro modelo es el } \\
\text { de municipio más } \\
\text { pequeño pero con } \\
\text { fuerte capacidad } \\
\text { como centro de } \\
\text { trabajo }\end{array}$ & $\begin{array}{l}\text { Aunque una mayoría } \\
\text { presenta dinámicas } \\
\text { demográficas } \\
\text { positivas no se } \\
\text { atisban procesos de } \\
\text { metropolitanización } \\
\text { reseñables, salvo } \\
\text { en la posible } \\
\text { coalescencia o } \\
\text { fusión con áreas } \\
\text { cercanas en los } \\
\text { casos de Pontevedra, } \\
\text { Ferrol y Benidorm. }\end{array}$ \\
\hline
\end{tabular}




\section{REGIONES METROPOLITANAS}

Con la denominación regiones metropolitanas se define una categoría de áreas metropolitanas que por su extensión espacial y complejidad funcional y estructural interna se sitúan en un nivel superior de organización y despliegue territorial de los procesos urbano-metropolitanos. En primer lugar, por su tamaño, que es fruto no de una referencia administrativa prevalente, sino de la aplicación de una metodología de delimitación, consistente y comparable internacionalmente, que nos ofrecen unas áreas muy extensas que albergan a varios millones de habitantes. Aunque la región metropolitana de Barcelona está más contenida espacialmente, en parte por razones orográficas, la de Madrid se extiende por más de 170 municipios pertenecientes a 5 provincias de tres comunidades autónomas distintas, desplegando casi 120.000 has de suelo artificializado de naturaleza urbana frente a los 70.000 de la región metropolitana de Barcelona. Ahora bien, la mera gran escala del fenómeno urbano no bastaría para su categorización como región metropolitana, si no se cuenta además con otros requisitos de organización funcional. Entre estos se encuentran la constitución, en el seno del conjunto del ámbito, de áreas y centros de organización funcional con un elevado grado de autonomía y complejidad funcional. Así, por ejemplo, ambas regiones metropolitanas incluyen en su seno otras "áreas metropolitanas" que cumplen los requisitos de delimitación establecidos. Concretamente, son los casos de Sabadell en la de Barcelona y Alcalá de Henares en Madrid que se incorporan o si se prefiere, son subsumidas por la ampliación del área principal. Pero junto a ello, y sobre todo, lo que aparece es un numeroso conjunto de áreas de funcionamiento diferenciado en las dos dimensiones que se han analizado: el de los mercados de trabajo y los de vivienda.

En cuanto a sus dinámicas internas lo que caracteriza a estas regiones metropolitanas, frente al resto del sistema metropolitano español, es en primer lugar la creciente importancia de los movimientos periféricos, que en coherencia con lo visto anteriormente presentan las tasas relativas más altas de otras todas las españolas. En segundo lugar la permanencia de los procesos de desconcentración, pero ya con menores intensidades relativas que en otras áreas de menor dimensión, aunque en valores absolutos todavía supongan flujos de dimensión relevante. En tercer lugar, la emergencia de flujos de recentralización significativos desde la primera corona metropolitana, todavía no perceptibles excesivamente en su impacto en valores globales, pero si cuando se cartografían singularmente sus flujos absolutos.

\section{III. ÁREAS METROPOLITANAS CONSOLIDADAS}

Por debajo de las regiones metropolitanas, en un segundo escalón de la jerarquía urbana, pero cuando el área metropolitana tiene una notable dimensión -en España ello puede situarse en torno al millón de habitantes- y ha alcanzado una madurez en su dinámica evolutiva, nos encontramos con lo que denominamos -siguiendo la terminología acuñada por la Oficina del Censo norteamericano- las áreas metropolitanas consolidadas.

Sin alcanzar las dimensiones y complejidad de las regiones metropolitanas, las áreas metropolitanas consolidadas se caracterizan igualmente, en su organización interna, por un papel activo del conjunto de la corona metropolitana, la cual, lejos de ser exclusivamente un mero receptor pasivo de la descentralización procedente de la ciudad central, presenta 
pautas autónomas relevantes de organización espacial, iniciando con ello la trayectoria hacía la constitución de la ciudad metropolitana, la cual se caracteriza -al igual que en la ciudad tradicional- porque los diversos componentes territoriales del área metropolitana se imbrican entre sí en las diferentes funciones y relaciones urbanas. Es en las áreas metropolitanas de Valencia, Sevilla y Bilbao donde claramente se manifiestan las características mencionadas de madurez y complejidad estructural. Las tres se refieren a áreas metropolitanas con más de un millón de habitantes, en los que además la corona supera en población a la ciudad central, lo que implica consiguientemente, de una parte, un consolidado proceso de movilidad residencial metropolitana y, de la otra, unos muy intensos vínculos de relación residenciatrabajo, entre los que se cuentan por decenas los flujos que superan el millar de trabajadores. Pero siendo ese último hecho significativo de por sí, lo relevante para caracterizar a estas áreas metropolitanas es la pauta de organización espacial de la movilidad residencia-trabajo, que ya no se restringe a la convencional bidireccional corona-centro, sino que afecta a sectores y espacios en el seno de la propia corona.

En definitiva, se trata de espacios metropolitanos plenamente consolidados en los que la ciudad central va cediendo protagonismo a las coronas metropolitanas. Estas, cada vez más, constituyen ámbitos autónomos de organización funcional y espacios con identidad propia que con el discurrir del tiempo se irán configurando como partes sustantivas de la nueva ciudad metropolitana con todos sus atributos, no sólo como meros contenedores de funciones especializadas, bien sea residenciales o productivas.

\section{IV. ÁREAS METROPOLITANAS POLINUCLEARES}

La lectura jerárquica de la taxonomía de las áreas metropolitanas españolas debe matizarse para introducir una categoría en la que el principio definitorio no son las características de organización espacial asociados al tamaño y dimensión del fenómeno metropolitano en cuestión, sino las derivadas de las particulares condiciones de la estructura territorial de asentamientos de partida. Esta aporta elementos de referencia que contribuyen a generar modelos de organización distintos de los monocéntricos convencionalmente descritos, en los que toda la estructura se arma a partir de una sola ciudad central que se expande por el territorio contiguo. Ahora bien, cuando un conjunto de ciudades relativamente cercanas tienen capacidad para desarrollar procesos metropolitanos, lo que puede producirse son formas de organización diferentes, que denominamos polinucleares, y que por otro lado no son fácilmente identificados por los métodos convencionales de delimitación de áreas como los aquí utilizados, basados en la movilidad residencia-trabajo. En efecto, en esta categoría se van a incluir sólo tres del total de la áreas metropolitanas españolas, de las que solo dos de ellas (Oviedo-Gijón-Avilés y Bahía de Cádiz-Jerez) pueden ser delimitadas con los criterios convencionales, mientras que la tercera (Málaga-Marbella) surge de la doble adscripción de dos municipios situadas entre ambas (Mijas y Fuengirola).

El elemento definitorio de esta categoría es lógicamente la existencia de dos o más de ciudades centrales que, cumpliendo individualmente los requisitos de tamaño e integración funcional especificados para tener sus propias áreas, estén a la vez integradas entre sí. En este caso, a diferencia de las regiones metropolitanas, donde la existencia en su seno de ámbitos de organización interna puede provenir de la propia dinámica de crecimiento de la corona 
metropolitana o de la absorción de áreas metropolitanas pequeñas, en las organizaciones polinucleares lo que existe es una fusión de áreas con complementariedades funcionales y/o territoriales, dando lugar a formas de organización complejas y muy diversas. En todo caso, por su propia configuración, se trata de áreas metropolitanas con un tamaño notable, tanto en términos de urbanización -entre 13.000 y 23.000 has; con mayor extensión que el área metropolitana consolidada de Bilbao-; como demográficamente, ocupando los puestos sexto, séptimo y décimo de la jerarquía metropolitana en esta dimensión, siendo por tanto componentes relevantes del sistema urbano español.

\section{V. ÁREAS METROPOLITANAS ESTANDAR}

La cuarta categoría de áreas metropolitanas a considerar es la que aquí denominamos estandar. La utilización de dicho término se basa en una doble razón. En primer lugar porque esta categoría es la que incluye un mayor número de áreas y en segundo lugar, y sobre todo, porque es la que en su pauta de organización y estructura se ajusta al modelo convencional metropolitano; es decir una ciudad central que es a la vez lugar de trabajo para un hinterland relativamente amplio y foco emisor de procesos de suburbanización de la población. En esta categoría se incluirían un total de 15 áreas metropolitanas españolas, con una población, actualmente, entre los 800.000 y los 350.000 habitantes y entre las 13.000 y 5000 has de suelos urbanizados.

Todas las ciudades centrales de esta categoría aparecen como un consolidado lugar de trabajo, pero lo que les otorga la condición a estos espacios como sustancialmente metropolitanos es el despliegue de procesos de suburbanización residencial de la población desde la ciudad central. De esta forma, los movimientos centro-corona (suburbanización) son los dominantes en el seno de estas áreas metropolitanas, dentro de unas tasas de movilidad residencial generalmente altas. Se trata además de un proceso de desconcentración casi exclusivamente residencial, en el que la ciudad central aparece como referente casi absoluto de los orígenes de la movilidad residencial y de los destinos de la movilidad residencia trabajo, dibujando de tal manera el modelo convencional o estandar que se atribuye a la organización metropolitana.

\section{VI. ÁREAS METROPOLITANAS MENORES}

Un grupo de nueve españolas, que cumplen plenamente los requisitos establecidos para su definición como metropolitanas a partir de los criterios de movilidad residencia-trabajo, se han categorizado como áreas metropolitanas menores en función de su dimensión como fenómeno urbano, que en definitiva condiciona algunos rasgos estructurales de su condición metropolitana, hasta el punto de que ninguna de ellas suele asociarse en la nomenclatura académica o institucional con dicho término.

Se trata de áreas con una población que oscila entre los umbrales inferior de 180.000 y superior de los 255.000 habitantes, con ciudades centrales que en ningún caso llegan a la primera de las cifras reseñadas, lo que implica una menor capacidad de generar procesos urbano-metropolitanos de gran alcance. Así, la no muy excesiva entidad general de los flujos, la dirección dominante de la movilidad residencia-trabajo en dirección a la ciudad 
central, el escaso tamaño y ritmo de crecimiento de las coronas, que indican unos procesos todavía mínimos de desarrollos residenciales metropolitanos, son las características mas significativas de estas áreas metropolitanas menores; que por otro lado muestran que, junto al grupo siguiente, y al menos en España, los procesos iniciales de metropolitanización están marcados, mucho antes que como mercados unitarios de vivienda, por su articulación como mercados de trabajo.

\section{VII. ÁREAS METROPOLITANAS INCIPIENTES}

La última categoría de áreas metropolitanas es la que se ha calificado como áreas metropolitanas incipientes, en la medida en que los procesos metropolitanos apenas aparecen dibujados tanto en lo se refiere a su organización funcional como a su materialización en la propia urbanización del territorio. Lo constituye un grupo relativamente numeroso en el que se incluyen un total de doce áreas con dos perfiles distintos. Uno, corresponde a un pequeño conjunto de cuatro áreas que, teniendo las características descritas en el grupo anterior, posee una aún menor dimensión poblacional y funcional, por lo que tanto sus niveles e intensidades de organización funcional, como la materialización de los procesos de urbanización metropolitanos son extraordinariamente limitados.

El segundo modelo de área metropolitana incipiente, el más común con ocho áreas, es el que responde a una ciudad central con un cierto tamaño de población y casi siempre con un extenso término municipal, mientras que la corona metropolitana queda reducida a una mínima parte del conjunto, representando menos del $10 \%$ de la población total del ámbito en la mayoría de estas áreas y en ninguno superando el 20\%. Todos ellos cuentan con un relativamente extenso término municipal, que hace que una gran parte de los procesos de crecimiento metropolitano y suburbanización residencial y de actividades se produzcan en el seno del mismo.

Estas circunstancias conducen a que sea muy difícilmente perceptible la condición metropolitana de los ámbitos incluidos en esta categoría, pertenezcan a un modelo de organización u otro. Por esta razón, solo en los casos de las áreas con ciudades de mayor tamaño o mediante procesos de coalescencia con otras mayores en las de pequeño tamaño pueden plantearse posibles evoluciones urbanas que hagan pasar de su actual condición de incipientes hacía modelos de organización plenamente metropolitanos.

\section{CONCLUSIONES}

La constatación de la condición de la actual ciudad en España como metropolitana no pasa de ser ya una afirmación incontestable. El hecho de que en la configuración territorialadministrativa y en las propias estadísticas públicas españolas apenas tenga reconocimiento esta condición, no es óbice para que desde la perspectiva científica sea relevante avanzar en el conocimiento de la definición, naturaleza y funcionamiento de esta realidad urbana.

Y una tarea necesaria es profundizar en la muy diferente naturaleza y funcionamiento que se esconde bajo el paraguas de la definición genérica de áreas metropolitanas, porque en esa diversidad se hallan algunas de las claves para entender la organización, estructuras y dinámicas de los procesos urbanos en España. 
Lo que se ha pretendido en este artículo es afrontar la tarea mencionada a través de la identificación de categorías dentro del conjunto de áreas metropolitanas españolas más allá de las simplistas establecidas por tamaño de población, sino que den cuenta de circunstancias distintivas, tanto en lo que se refiere tanto a su naturaleza como a su funcionamiento, y que puedan constituir un primer paso para entender y reconocer con mayor precisión el fenómeno metropolitano en España, desde nuestras ciudades globales hasta los incipientes y a veces inadvertidos espacios metropolitanos.

En cualquier caso, tanto la aproximación realizada como la caracterización y los elementos y variables aquí elegidas, entre otras muchas posibles, se aportan en este artículo como una contribución abierta y sometida a su necesaria discusión y mejora para avanzar en el adecuado entendimiento y explicación de la actual geografía urbana española. 